ORIGINAL PAPER

\title{
Histopathological LIVER Findings in PATIENTS WITH HEPATOCEREBRAL MITOCHONDRIAL DEPLETION SYNDROME WITH DEFINED MOLECULAR BASIS
}

\author{
Maciej Pronicki ${ }^{1}$, Dorota Piekutowska-Abramczuk ${ }^{2}$, Dariusz Rokicki ${ }^{3}$, \\ Katarzyna Iwanicka-Pronicka ${ }^{2,4}$, WiesŁawa Grajkowska ${ }^{1}$
}

\begin{abstract}
${ }^{1}$ Department of Pathology, The Children's Memorial Health Institute, Warsaw, Poland ${ }^{2}$ Department of Genetics, The Children's Memorial Health Institute, Warsaw, Poland ${ }^{3}$ Department of Paediatrics Nutrition, and Metabolic Disorders, The Children's Memorial Health Institute, Warsaw, Poland ${ }^{4}$ Department of Audiology and Phoniatrics, The Children's Memorial Health Institute, Warsaw, Poland
\end{abstract}

\begin{abstract}
Mitochondrial DNA depletion consisting of the systemic reduction of mtDNA copy number in cells may have a heterogenous genetic basis, resulting from a pathogenic change in the nuclear genes involved in mtDNA synthesis. The mode of inheritance is autosomal recessive. Severe hepatocerebral disease represents one of many different clinical forms of so-called mitochondrial depletion syndrome (MDS). We present the liver histopathology of 13 children who eventually died in the course of hepatocerebral MDS confirmed molecularly, harbouring mutations of DGUOK, $M P V 17$, and POLG genes. Material comprising eight autopsy and five liver biopsy specimens showed a moderately reproducible pattern of parenchymal damage, which we consider potentially helpful in the differential diagnosis and planning of the diagnostic investigation in families of children who died due to early-onset acute liver failure and encephalopathy.
\end{abstract}

Key words: mitochondrial DNA depletion, liver failure, POLG1, DGUOK, MPV17.

\section{Introduction}

In 1991 the first infants were described in whom a fatal mitochondrial disease was associated with very low copy number of mitochondrial DNA (mtDNA) in specific tissues [1]. Subsequently, several cases of mitochondrial depletion were reported in infants who died due to acute liver failure [2]. Now we know that the mitochondrial depletion syndrome (MDS) may present hepatocerebral, encephalomyopathic, myopathic, and neurogastrointestinal forms, and is caused by different gene defects [3]. The nuclear genes responsible for appropriate mitochondrial DNA synthesis act at different points. In general, they may regulate either mtDNA polymerisation
(POLG) or control the supply of deoxyribonucleoside triphosphates indispensable for mtDNA synthesis (DGUOK, MPV17). The hepatocerebral form of MDS in Poland is an extremely rare condition. Moreover, our observation indicates that a low copy number of mtDNA may occur also in non-genetic liver failure, and this parameter should be considered in relation to histopathological features of MDS [4].

The aim of the study is to characterise the histopathological picture of the liver in the differential diagnosis of hepatic depletion syndromes in the clinical setting of acute liver failure in children. It has not been analysed for MDS as a whole, with the exception of Alpers-Huttenlocher syndrome caused by POLG mutations [5, 6]. 


\section{Material and methods}

Thirteen patients with hepatocerebral MDS with confirmed molecular defects in POLG, DGOUG, or $M P V 17$ genes were included in the examined group.

Histopathological study comprised five core liver biopsies and eight liver autopsy samples (one test of each patient).

Tissue samples were formalin fixed and paraffin embedded. The panel of histological stains included haematoxylin and eosin, PAS, PAS after diastase digestion, reticulin stain, and AZAN stain for fibrosis evaluation.

The study was conducted in accordance with the principles outlined in the Declaration of Helsinki. All parents or authorised guardians of the patients gave their informed consent for the study. The protocol was approved by the CMHI Bioethics Commission.

\section{Histopathological evaluation criteria}

For the purpose of the present study, we discerned different patterns of liver injury and applied the following grading/staging criteria for liver parenchymal damage and transformation.

1. Diffuse post-necrotic fibrosis with neocholangiolisation $(0$ - absent; 1 - present $)$.

This pattern of liver damage appears to be easy recognisable. Diffuse fibrosis with numerous proliferating neocholangioles replaces damaged liver parenchymal cells. There is almost no tendency and/ or potential for liver cell regeneration and nodular transformation. Scant preserved hepatocytes usually show advanced degenerative changes, microvesicu-

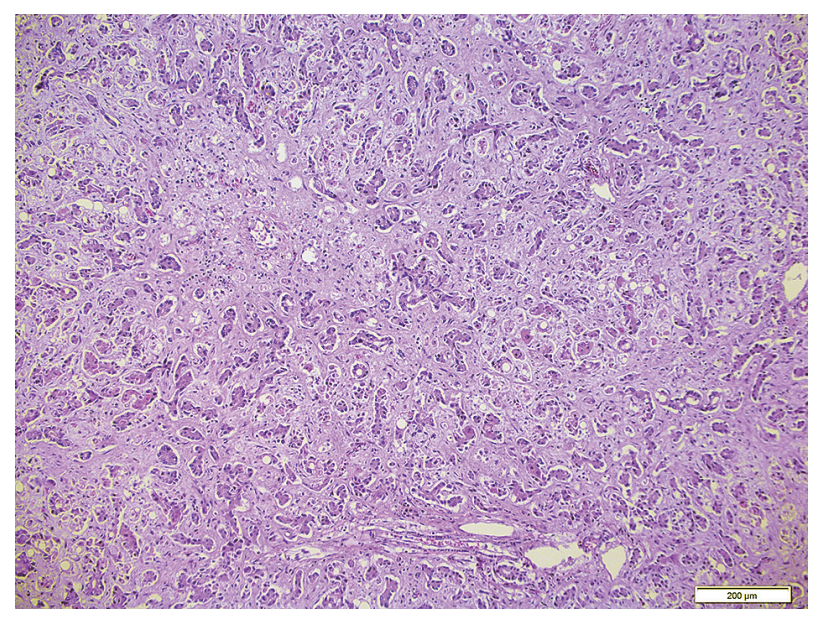

Fig. 1. Histopathological liver pattern of MSD caused by POLG gene defect (patient 7, autopsy). Almost total hepatocyte loss and replacement by diffuse fibrosis with numerous neocholangiolar profiles. Original magnification $100 \times$, HE stain. This pattern was seen in all patients with $P O L G$ gene mutations lar steatosis, and severe cholestasis. This pattern, if present, usually presents the end stage form of organ damage and has not been graded in this study (Figs. 1-3).

2. Fibrosis leading to nodular transformation/cirrhosis (0-4 Batts and Ludwig) [7] (Fig. 4).

Fibrosis progressing from portal involvement through fibrous bridging to the development of cirrhosis, being the most typical sequence of damaged liver transformation, has been staged according to the five-degree Batts and Ludwig system (0 to 4) conceived for evaluation of necroinflammatory liver injury and fibrosis in hepatitis [7]. This scale was adopted due to its simplicity and reproducibility, as well as possible practical usage also in non-inflammatory liver disorders. Due to conceptually different patterns of liver fibrous transformation categories 1 and 2 (diffuse vs. nodular) are recorded as mutually exclusive, although some degree of overlap sometimes may exist.

3. Portal inflammation.

4. Lobular inflammation.

Portal and lobular inflammation have been graded according to five-point system (0 to 4 ), as used for staging of inflammation in hepatitis in the Batts and Ludwig scale [7].

5. Microvesicular steatosis (0-3) none; mild; moderate; severe.

6. Macrovesicular steatosis (0-3) none; mild; moderate; severe.

Steatosis, macro-, microvesicular, or mixed has been graded as: absent, mild, moderate or severe, depending on liver parenchymal area with lipid accumulation $(0-5 \%$; 6-30\%; 31-70\%; and $71-100 \%$, respectively).

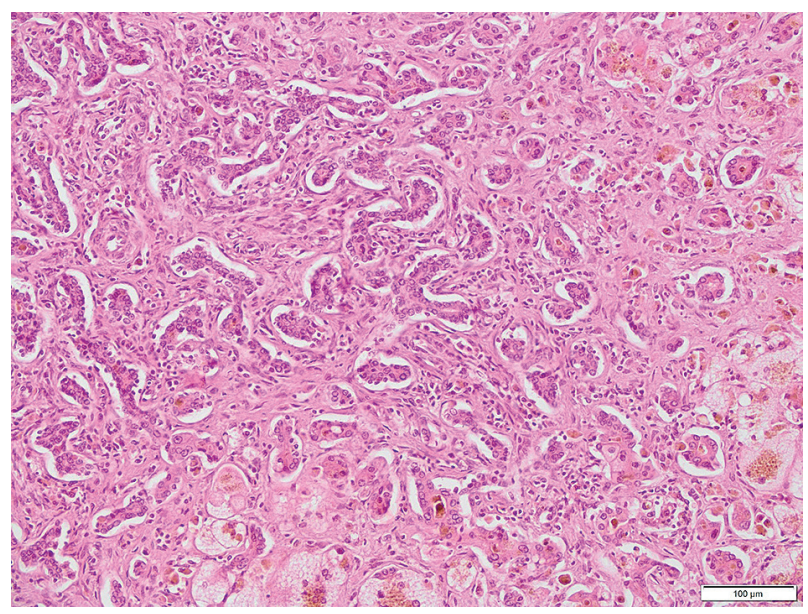

Fig. 2. Histopathological liver pattern of MSD caused by $D G U O K$ gene defect (patient 11, autopsy). Typical scarring pattern with small residual foci of hepatocytes with advanced degeneration (lower edge and right corner). Original magnification $200 \times$, HE stain 


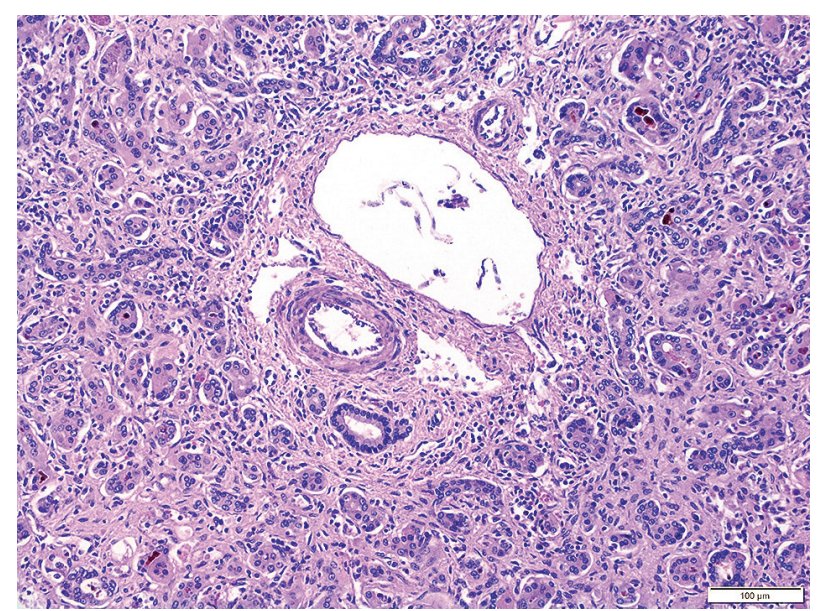

Fig. 3. Histopathological liver pattern of MSD caused by DGUOK gene defect (patient 10, autopsy). Portal tract with total loss of surrounding hepatocytes, replaced by fibrous tissue with cholangiolar structures. Original magnification $200 \times$, HE stain

7. Cholestasis (0-3) none; mild; moderate; severe.

Cholestasis has been assessed subjectively and graded from absent to severe using semi-quantitative 4-point scale (0 to 3).

8. Recent "fresh" necrosis.

Recent necrosis was recorded when the areas of liver cell damage had not been replaced by post necrotic fibrous scar or proliferating neocholangioles and graded semi-quantitatively in four-point scale.

9. Liver cell degeneration and ballooning (intensity 0 to 3 ).

Collective analysis of histopathological changes was carried out on the basis of a common questionnaire developed for the needs of this work.

\section{Molecular methods}

Total DNA was isolated from available tissues (blood, muscle, liver) by standard extraction methods (phenol/chloroform or automatic MagNA Pure LC 2.0, Roche). The mutation analysis was performed with the use of Sanger sequencing of PCR amplified exons with exon/intron boundaries of the MPV17, $P O L G$, and DGUOK genes. Numbering of revealed nucleotide changes was based on reference sequences for the MPV17 (RefSeq: NM 002437.4 NP 002428.1), POLG (RefSeq: NM_002693.2; NP-002684.1), and DGUOK genes (RefSeq: NM_-080916.2; NP_550438.1).

\section{Results and comments}

\section{Clinical and molecular characteristics}

The course of the disease and the molecular basis are presented in Table I. In patients with DGUOK

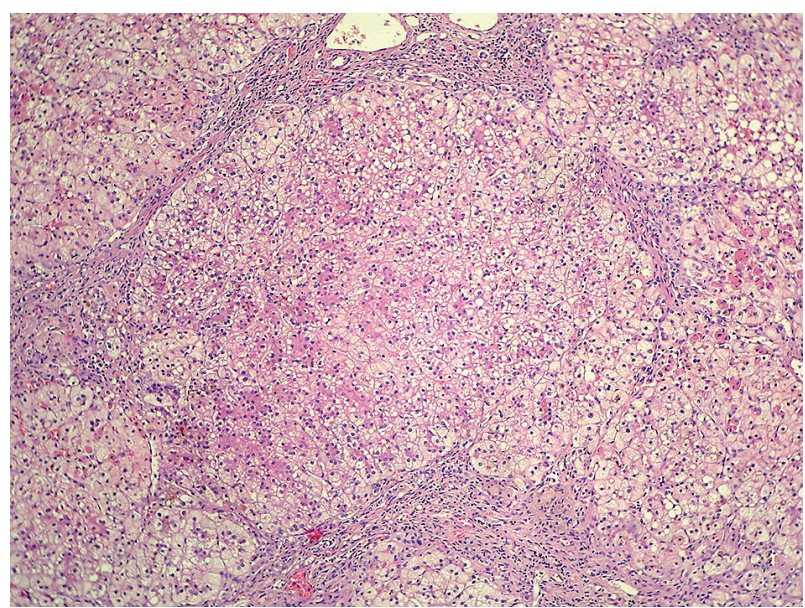

Fig. 4. Histopathological liver pattern of MSD caused by MPV17 gene defect (patient 1, autopsy). Classical nodular transformation with hepatocyte necrosis, degeneration, and steatosis. Original magnification $200 \times$, HE stain. This, or similar pattern, was seen in patients with MPV17 gene mutations

and MPV17 defects, hepatic symptoms dominated; neurological symptoms occurred later and were mild. In patients with POLG defect, severe epilepsy prevailed at the beginning, and liver failure did not appear until valproate administration. All patients died from hepatic insufficiency aged 2 to 42 months, several while waiting for liver transplantation. In all subjects, the mtDNA/nDNA ratio in the liver was extremely low $(0.01-11 \%$ of the reference values). Case reports of part of the patients have been previously described in the literature. Relevant references are given in Table I.

Results of molecular analysis of MPV17, DGUOK, and POLG are summarised in Table I. The above-mentioned genes are involved in mtDNA replication and have been shown to cause MDS as well as multiple deletions, resulting in infantile or later-onset mitochondrial diseases.

Our experience so far indicates that molecular diagnostics for MDS should rely primarily on the exclusion of DGUOK, MPV17, and POLG mutations, in particular the common molecular variants, and then in the case of a negative result in further diagnostics.

\section{Histopathological studies}

Based on the assessment of five biopsies and eight hepatic sections from patients with known hepatocerebral depletion syndromes (DGUOK, $M P V 17, P O L G)$, the diagnostic value of individual histopathological parameters was estimated and the "pattern" of these changes corresponding to the diagnosis of MDS was determined (Table II). It is thoroughly described and discussed in the next section. 


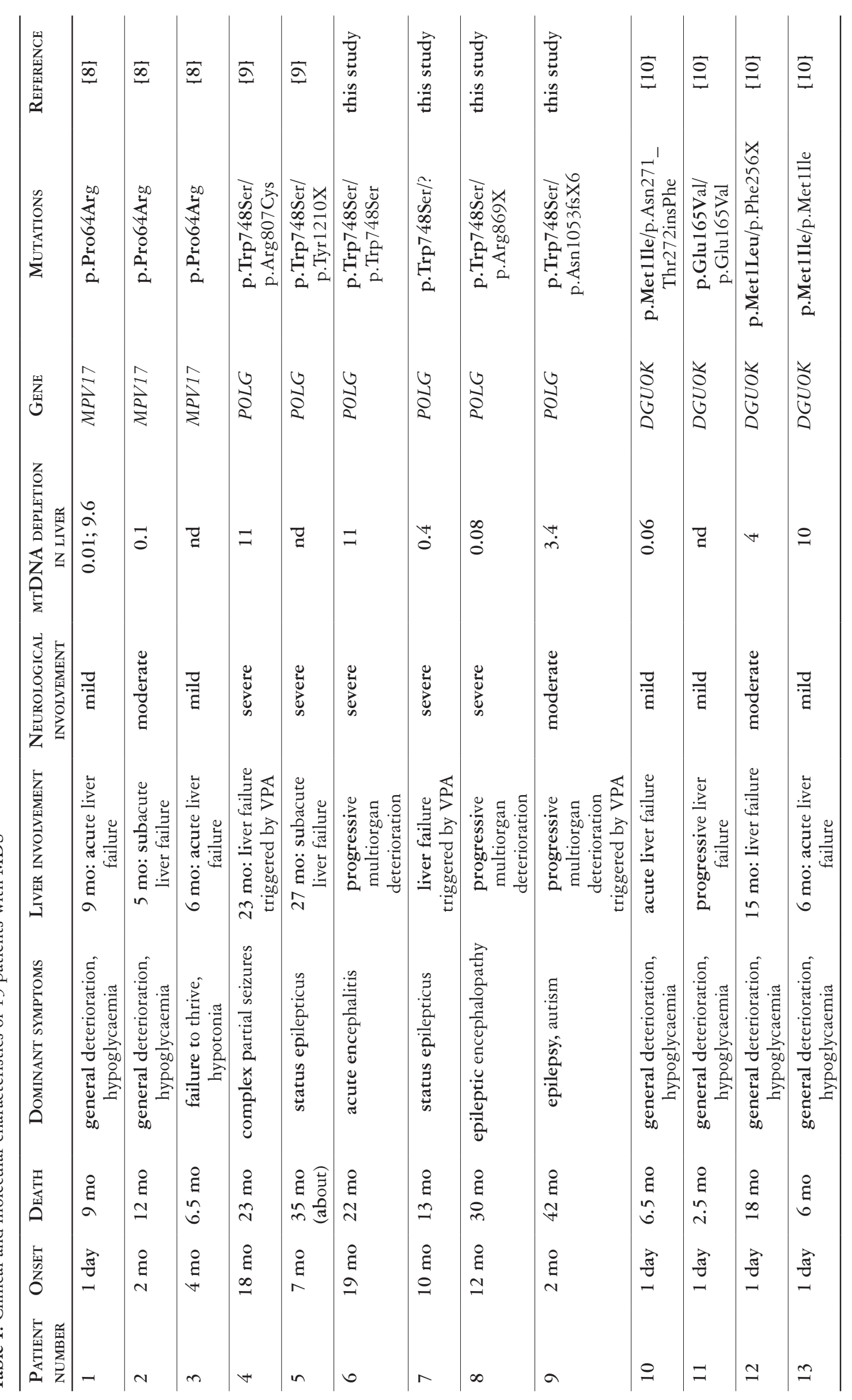




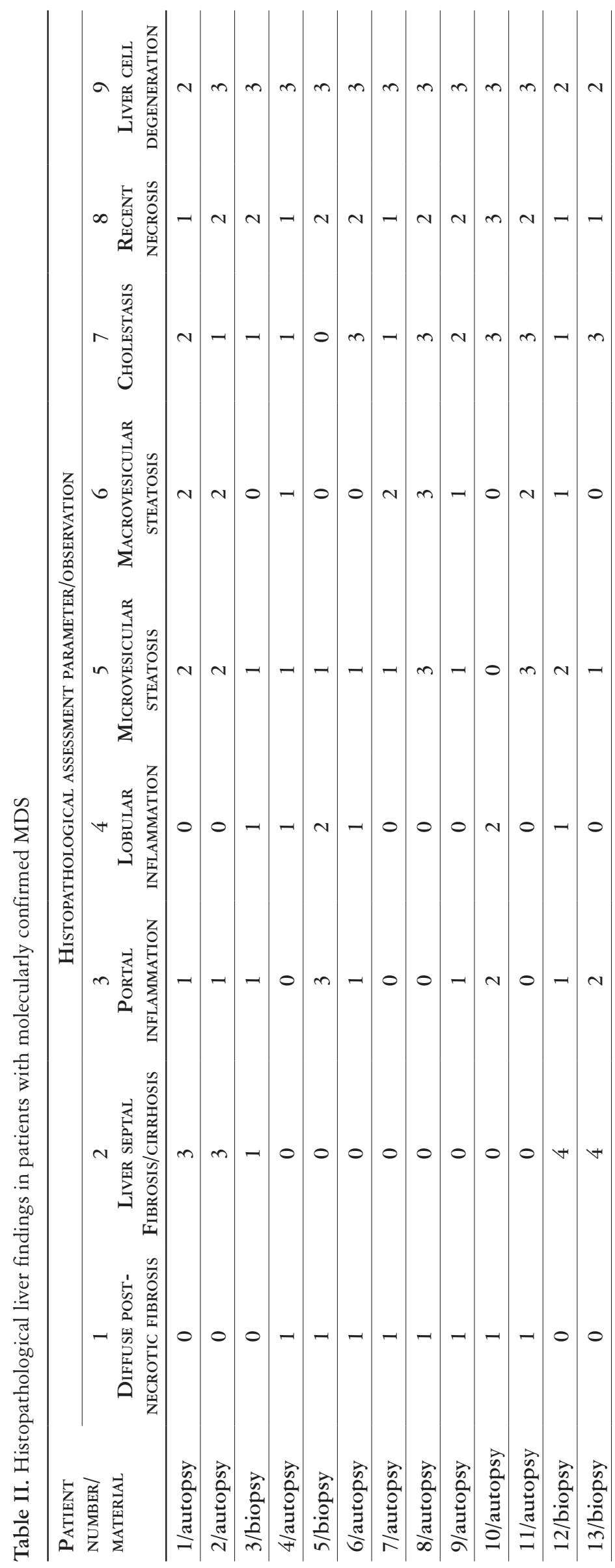




\section{Discussion}

The aim of this study was to call histopathologists' attention to MDS as one of the possible causes of an acute liver failure in neonates, infants, and young children, and to summarise liver histopathological findings. Clinically hepatic involvement is usually accompanied by some degree of neurological dysfunction and features of encephalopathy. In the majority of patients with the fully developed stage of the disease it may be difficult to recognise encephalopathy as a separate, inherent feature of MDS, because it may be considered secondary to (usually severe) liver dysfunction. In all children from our study group, the liver damage was severe, eventually leading to the patient's death. It is obvious that autopsy material reveals more advanced liver injury than biopsy samples because indications for biopsy procedure require adequate liver sufficiency, mainly proper synthesis of coagulation factors.

In our opinion, the pattern of diffuse postnecrotic fibrosis with neocholangiole formation, reflecting massive parenchymal damage and scarring, consists of a common end point in the majority of patients (patients 4-11). It may be accompanied by variable degrees of degeneration, steatosis, and cholestasis of residual hepatocytes. The foci of surviving hepatocytes are larger at the early stages of liver disease progression, giving the impression of nodular transformation and retained capacity for regeneration. Understandably, this pattern is more likely to be seen in biopsy material (patients 12 and 13). In fact, knowing the primary cause of disease, the fate of liver parenchymal cells is invariably unfortunate. In some patients diffuse fibrosis is not so pronounced, and nodular transformation with mixed steatosis and degeneration predominates (patients 1, 2, 3).

In a few publications similar liver lesions in MDS have been reported by other authors. Morris et al. described "geographic areas of liver fatty change, bile duct proliferation, collapse of liver architecture and fibrosis" in the biopsy and autopsy material of three patients with mtDNA depletion [2]. They stated that liver histology can be helpful in making the diagnosis of MDS. Here, it must be clarified that in MDS "bile duct proliferation" should be described as "diffuse neocholangiole formation and fibrosis" because the first term is widely understood as ductular reaction at the periphery and/or inside portal tracts in liver disease (e.g. in biliary atresia), while the second is the reflection of diffuse irreversible hepatocyte damage and replacement fibrous-biliary scarring. Regardless of the name, the pattern of liver damage in Morris' study microphotographs was the same as that observed by us in advanced post-mortem cases [2]. Similar hepatic lesions were also comprehensively presented by Harding, who described them in autopsy livers of 26/30 patients with Alpers Huttenlocher syndrome, calling them "typical". The remaining four patients showed mid-zonal necrosis or fatty change only [11].

The child who succumbed to progressive disease with features of liver failure may pose a diagnostic challenge for pathologist in post-mortem examination. In our experience, in some patients the superimposed features of infection and sepsis may be misleading in establishing the primary cause of fatal disease because the liver degenerative and post-necrotic lesions may be falsely interpreted as a secondary phenomenon. It should be emphasised that since in hepatocerebral MDS the clinical course may by rapidly fatal, the autopsy may consist of the last opportunity for obtaining diagnostic material for molecular investigation. Thorough neuropathological examination is of course mandatory because it may potentially reveal helpful findings.

The role of molecular assessment in the whole investigation is conclusive, but the strategy of the diagnostic approach should take advantage of all pathological observations. For the present, we believe that the histopathological pattern may suggest a molecular background in POLG and DGUOK mutations, which leads to the development of "typical" diffuse scarring and neocholangliolisation (Figs. 1-3). This concerns late stage of liver damage, as has already been mentioned. Patients with MPV17 mutations, and DGUOK at an early stage, show classical cirrhotic pattern with prominent steatosis (Fig. 4). The latter pattern of liver damage is generally non-specific, so its diagnostic usefulness seems disputable.

Another issue that should be mentioned is the secondary mtDNA depletion, which may be found in tissues with pathological architectural or necrotic transformation leading to alteration of nDNA to mtDNA proportion. Interestingly, in our previous study, in depleted livers in which the molecular testing was negative, the liver histopathology picture had a different character. Among others, extensive haemorrhagic necrosis, inflammatory features, or other non-specific lesions were seen. Negative correlation was found in the total 243 liver specimens between the value of the mtDNA/nDNA ratio and the presence of fibrosis, inflammation, and necrosis [4].

\section{Conclusions}

The possibility of MDS should be considered in all children with early-onset acute liver failure, especially if accompanied by neurological dysfunction. In hepatocerebral MDS, fully developed liver disease presents a reproducible pathological pattern of parenchymal damage. The value of histopathological examination in differential diagnosis of MDS is supplementary to the analysis of mtDNA depletion in 
tissues. Due to the possibility of secondary mtDNA depletion, both of these parameters must be analysed together (equally).

\section{Acknowledgements}

Molecular data presented in Table I were partially prepared by Katarzyna Tońska MD, and Katarzyna Strawa from Warsaw Medical University, Poland and Maria Tesarova from Karol University, Prague, Czech Republic.

This study was partially supported by grants from the Polish National Science Centre No. N 2857/B/ P01/2010/39, 2012/05/B/NZ2/01627.

\section{References}

1. Moraes CT, Shanske S, Tritschler HJ, et al. mtDNA depletion with variable tissue expression: a novel genetic abnormality in mitochondrial diseases. Am J Hum Genet 1991; 48: 492-501.

2. Morris AA, Taanman JW, Blake J, et al. Liver failure associated with mitochondrial DNA depletion. J Hepatol 1998; 28 : 556-563.

3. El-Hattab AW, Scaglia F, Craigen WJ, et al. MPV17-Related hepatocerebral mitochondrial DNA depletion syndrome. In: GeneReviews ${ }^{\circledR}$ [Internet $]$. Adam MP, Ardinger HH, Pagon RA, et al. (eds.). University of Washington, Seattle 19932018.

4. Piekutowska-Abramczuk D, Pronicki M, et al. Hepatocerebral depletion syndrome in children - methodology, study results and recommendations. Standardy Medyczne Pediatria 2016; 13: 869-876.

5. Huttenlocher PR, Solitare GB, Adams G. Infantile diffuse cerebral degeneration with hepatic cirrhosis. Arch Neurol 1976; 33: $186-192$

6. Naviaux RK, Nguyen KV. POLG mutations associated with Alpers' syndrome and mitochondrial DNA depletion. Ann Neurol 2004; 55: 706-712.

7. Batts KP, Ludwig J. Chronic hepatitis. An update on terminology and reporting. Am J Surg Pathol 1995; 19: 1409-1417.

8. Piekutowska-Abramczuk D, Pronicki M, Strawa K, et al. Novel c.191C $>$ G (p.Pro64Arg) MPV17 mutation identified in two pairs of unrelated Polish siblings with mitochondrial hepatoencephalopathy. Clin Genet 2014; 85: 573-577.

9. Pronicka E, Węglewska-Jurkiewicz A, Pronicki M, et al. Drugresistant epilepsia and fulminant valproate liver toxicity. Alpers-Huttenlocher syndrome in two children confirmed post mortem by identification of p.W748S mutation in POLG gene. Med Sci Monit 2011; 17: CR203-209.

10. Pronicka E, Węglewska-Jurkiewicz A, Taybert J, et al. Post mortem identification of deoxyguanosine kinase (DGUOK) gene mutations combined with impaired glucose homeostasis and iron overload features in four infants with severe progressive liver failure. J Appl Genet 2011; 52: 61-66.

11. Harding BN. Progressive neuronal degeneration of childhood with liver disease (Alpers Huttenlocher syndrome). A personal review. J Child Neurol 1990; 5: 273-287.

\section{Address for correspondence}

Wiesława Grajkowska

Department of Pathology

The Children's Memorial Health Institute

Aleja Dzieci Polskich 20

04-730 Warsaw, Poland

tel. +48228151960

fax +48228151975

e-mail: w.grajkowska@ipczd.pl 